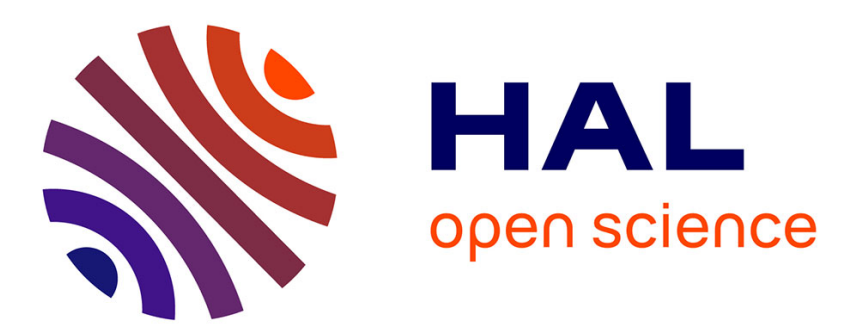

\title{
Migration of double imaginary characteristic roots under small deviation of two delay parameters
}

\author{
Keqin Gu, Dina Irofti, Islam Boussaada, Silviu-Iulian Niculescu
}

\section{To cite this version:}

Keqin Gu, Dina Irofti, Islam Boussaada, Silviu-Iulian Niculescu. Migration of double imaginary characteristic roots under small deviation of two delay parameters. 54th IEEE Conference on Decision and Control (CDC), Dec 2015, Osaka, Japan. 10.1109/cdc.2015.7403229 . hal-01261215

\section{HAL Id: hal-01261215 \\ https://hal.science/hal-01261215}

Submitted on 24 Jan 2016

HAL is a multi-disciplinary open access archive for the deposit and dissemination of scientific research documents, whether they are published or not. The documents may come from teaching and research institutions in France or abroad, or from public or private research centers.
L'archive ouverte pluridisciplinaire HAL, est destinée au dépôt et à la diffusion de documents scientifiques de niveau recherche, publiés ou non, émanant des établissements d'enseignement et de recherche français ou étrangers, des laboratoires publics ou privés. 


\title{
Migration of double imaginary characteristic roots under small deviation of two delay parameters
}

\author{
Keqin $\mathrm{Gu}^{1}$ \\ Dina Irofti ${ }^{2}$ \\ Islam Boussaada ${ }^{2}$ \\ Silviu-Iulian Niculescu ${ }^{2}$
}

\begin{abstract}
This paper studies the migration of double imaginary roots of the characteristic equation for systems with two delays when the delay parameters are subjected to small deviations. As the double roots are not differentiable with respect to the delay parameters, Puiseux series is often used in such a situation in the literature. In this article, we study the "least degenerate" case, and a more traditional analysis was used without Puiseux series. It was found that the local stability crossing curve has a cusp at the point in the parameter space that causes the double root, and it divides the neighborhood of this point into a G-sector and an S-sector. When the parameters move into the G-sector, one of the roots moves to the right half plane, and the other moves to the left half plane. When the parameters move into the $S$-sector, both roots move either to the left half plane or the right half plane depending on the sign of some value explicitly expressed in terms of derivatives of the characteristic function up to the third order.
\end{abstract}

\section{INTRODUCTION}

An effective approach of stability analysis is Ddecomposition (also known as D-partitioning or Dsubdivision) method [6]. The method is especially important for systems with time-delays, as shown in [1] [4] due to difficulty of direct analysis for such systems. The main idea of this method is to first identify the stability crossing set, which divides the parameter space into regions, each of which has a constant number of right half plane characteristic roots. The particular case where these parameters are the delays has been called $\tau$-decomposition method by Lee and Hsu [13] (see also [15]).

Most analysis in the literature discusses only the nondegenerate cases. One degenerate case often excluded from discussion is when there exist multiple imaginary roots for some parameter values (see [10], [14], [7]). To be specific, consider the system with characteristic equation

$$
p\left(s, \tau_{1}, \tau_{2}\right)=p_{0}(s)+p_{1}(s) e^{-\tau_{1} s}+p_{2}(s) e^{-\tau_{2} s}=0,
$$

with two delays as the parameters discussed in [7]. Then the stability crossing set consists of curves in the $\tau_{1}-\tau_{2}$ parameter space. If $p\left(s, \tau_{10}, \tau_{20}\right)$ has a double root at $j \omega_{0}$, then the stability crossing set has a cusp at $\left(\tau_{10}, \tau_{20}\right)$. Such nonsmoothness means that conventional analysis based on the first-order derivatives no longer applies. Indeed, " $s$ " as an implicit function of $\tau_{1}$ and $\tau_{2}$ defined by (1) is no longer differentiable at $s=s_{0}=j \omega_{0}$ and multivalued in its neighborhood.

It has long been recognized that the roots of a polynomial are continuous functions of coefficients as long as the leading

\footnotetext{
${ }^{1}$ Keqin Gu is with Department of Mechanical and Industrial Engineering, Southern Illinois University Edwardsville, Edwardsville, Illinois 62026, USA. kgu@siue.edu

${ }^{2}$ Dina Irofti, Islam Boussaada, and Silviu-Iulian Niculescu are with Laboratoire des Signaux et Systèmes (L2S) SupélecCNRS-Université Paris Sud, 3 rue Joliot-Curie 91192 Gif-surYvette cedex, France. Dina.Irofti, Islam.Boussaada, Silviu.Niculesculls. centralesupelec.fr
}

coefficient does not vanish [12]. Furthermore, these functions are differentiable in the case of simple roots. In the case of multiple roots, differentiability is lost, and Puiseux series may be used to analyze such cases (see [11] and Part II, Chapter 5 of [12]). These conclusions are also valid for time-delay systems of retarded type, and analysis based on Puiseux series for time-delay systems can be found [2] [3] [14].

In this article, we will study the case of double characteristic imaginary roots when it is the "least degenerate", and show that a more conventional method without invoking Puiseux series is still possible. A simple condition is derived regarding how the double characteristic roots migrate as the delay parameters deviate from the critical value $\left(\tau_{10}, \tau_{20}\right)$.

\section{Problem statement}

Consider a system with (1) as the characteristic equation, where $p_{k}(s), k=0,1,2$ are polynomials of " $s$ " with real coefficients. For $\tau_{1}=\tau_{10}, \tau_{2}=\tau_{20}$, we assume $p\left(s, \tau_{1}, \tau_{2}\right)$ has a double root on the imaginary axis $s=s_{0}=i \omega_{0}$. More precisely, we assume

$$
\begin{gathered}
p\left(s_{0}, \tau_{10}, \tau_{20}\right)=0, \\
\left.\frac{\partial p}{\partial s}\right|_{\substack{s=s_{0} \\
\tau_{1}=\tau_{10} \\
\tau_{2}=\tau_{20}}}=0, \\
\left.\frac{\partial^{2} p}{\partial s^{2}}\right|_{\substack{s=s_{0} \\
\tau_{1}=\tau_{10} \\
\tau_{2}=\tau_{20}}} ^{\substack{s \\
\tau_{2}}} .0 .
\end{gathered}
$$

Furthermore, we will assume

$$
D=\Im\left(\frac{\partial p^{*}}{\partial \tau_{1}} \cdot \frac{\partial p}{\partial \tau_{2}}\right)_{\substack{s=s_{0} \\ \tau_{1}=\tau_{10} \\ \tau_{2}=\tau_{20}}} \neq 0,
$$

where $\Im(\cdot)$ denotes the imaginary part, and $(\cdot)^{*}$ denotes the complex conjugate of a complex number. We will also use $\Re(\cdot)$ to denote the real part. It is not difficult to see that $D$ may also be expressed as

$$
D=\operatorname{det}\left(\begin{array}{cc}
\Re\left(\frac{\partial p}{\partial \tau_{1}}\right) \Re\left(\frac{\partial p}{\partial \tau_{2}}\right) \\
\Im\left(\frac{\partial p}{\partial \tau_{1}}\right) \Im\left(\frac{\partial p}{\partial \tau_{2}}\right)
\end{array}\right) \begin{gathered}
\substack{s=s_{0} \\
\tau_{1}=\tau_{10} \\
\tau_{2}=\tau_{20}} \\
.
\end{gathered}
$$

Satisfaction of (2)-(5) will be the standing assumptions in this article, and a system satisfying these equations will be known as the least degenerate. Indeed, in view of (6), it can be seen that (5) implies that the characteristic equation (1) defines $\left(\tau_{1}, \tau_{2}\right)$ in a small neighborhood of $\left(\tau_{10}, \tau_{20}\right)$ as a function of $s$ in a sufficiently small neighborhood of $s_{0}$ in view of the implicit function theorem. Introduce the notation

$$
\begin{aligned}
\mathcal{N}_{\varepsilon}\left(x_{0}\right) & =\left\{x|| x-x_{0} \mid<\varepsilon\right\}, \\
\mathcal{N}_{\varepsilon}^{\circ}\left(x_{0}\right) & =\left\{x|0<| x-x_{0} \mid<\varepsilon\right\},
\end{aligned}
$$


then the above can be more precisely stated as follows.

Proposition 1: There exists a $\varepsilon>0$ and a sufficiently small $\delta>0$ such that for all $s \in \mathcal{N}_{\delta}\left(s_{0}\right)$, we may define $\tau_{1}(s)$ and $\tau_{2}(s)$ as the unique solution of (1) with $\left(\tau_{1}(s), \tau_{2}(s)\right) \in \mathcal{N}_{\varepsilon}\left(\tau_{10}, \tau_{20}\right)$. The functions so defined are differentiable to an arbitrary order.

It should be pointed out that in general, (1) may have other solutions outside of $\mathcal{N}_{\varepsilon}\left(\tau_{10}, \tau_{20}\right)$.

The set

$$
\begin{aligned}
& \mathcal{T}_{\left(\omega_{0}, \tau_{10}, \tau_{20}\right)}= \\
& \quad\left\{\left(\tau_{1}(i \omega), \tau_{2}(i \omega)\right) \in \mathcal{N}_{\varepsilon}\left(\tau_{10}, \tau_{20}\right) \mid i \omega \in \mathcal{N}_{\delta}\left(i \omega_{0}\right)\right\}
\end{aligned}
$$

represents a curve in the $\tau_{1}-\tau_{2}$ space that passes through the point $\left(\tau_{10}, \tau_{20}\right)$, and is the restriction of stability crossing curves $\mathcal{T}$ defined in [7] in a neighborhood of $\left(\tau_{10}, \tau_{20}\right)$. Therefore, $\mathcal{T}_{\left(s_{0}, \tau_{10}, \tau_{20}\right)}$ will be known as the local stability crossing curve. We will also denote

$$
\begin{aligned}
& \mathcal{T}_{\left(\omega_{0}, \tau_{10}, \tau_{20}\right)}^{+}= \\
& \left\{\left(\tau_{1}(i \omega), \tau_{2}(i \omega)\right) \in \mathcal{N}_{\varepsilon}\left(\tau_{10}, \tau_{20}\right) \mid i \omega \in \mathcal{N}_{\delta}\left(i \omega_{0}\right), \omega>\omega_{0},\right\}
\end{aligned}
$$

and

$$
\begin{aligned}
& \mathcal{T}_{\left(\omega_{0}, \tau_{10}, \tau_{20}\right)}^{-}= \\
& \left\{\left(\tau_{1}(i \omega), \tau_{2}(i \omega)\right) \in \mathcal{N}_{\varepsilon}\left(\tau_{10}, \tau_{20}\right) \mid i \omega \in \mathcal{N}_{\delta}\left(i \omega_{0}\right), \omega<\omega_{0}\right\} .
\end{aligned}
$$

The curves $\mathcal{T}_{\left(\omega_{0}, \tau_{10}, \tau_{20}\right)}^{+}$and $\mathcal{T}_{\left(\omega_{0}, \tau_{10}, \tau_{20}\right)}^{-}$will be known as the positive and negative local stability crossing curves, respectively.

The purpose of this article is to study how these two characteristic roots migrate as $\left(\tau_{1}, \tau_{2}\right)$ varies in a small neighborhood of $\left(\tau_{10}, \tau_{20}\right)$ in the least degenerate case.

\section{CUSP AND LOCAL BIJECTION}

Let

$$
s=s_{0}+u e^{i \theta} .
$$

Then $u$ and $\theta$ parameterize a neighborhood of $s_{0}$, and $\tau_{1}$ and $\tau_{2}$ can be considered as functions of $u$ and $\theta$. For the sake of convenience, write

$$
\gamma=e^{i \theta}=\frac{\partial s}{\partial u}
$$

We first fix the angular variable $\theta$, i.e., fix $\gamma$, and calculate the derivatives of $\tau_{1}$ and $\tau_{2}$ with respect to the radial variable $u$. This can be easily achieved by differentiating (1), yielding

$$
\frac{\partial p}{\partial \tau_{1}} \frac{\partial \tau_{1}}{\partial u}+\frac{\partial p}{\partial \tau_{2}} \frac{\partial \tau_{2}}{\partial u}+\frac{\partial p}{\partial s} \gamma=0
$$

Setting $u=0$ and using (3) in (9), we obtain

$$
\left(\begin{array}{ll}
\Re\left(\frac{\partial p}{\partial \tau_{1}}\right) & \Re\left(\frac{\partial p}{\partial \tau_{2}}\right) \\
\Im\left(\begin{array}{l}
\partial p \\
\partial \tau_{1}
\end{array}\right) & \Im\left(\frac{\partial p}{\partial \tau_{2}}\right)
\end{array}\right) \underbrace{}_{\substack{s=s_{0} \\
\tau_{1}=\tau_{10} \\
\tau_{2}=\tau_{20}}}\left(\begin{array}{l}
\frac{\partial \tau_{1}}{\partial u} \\
\frac{\partial \tau_{2}}{\partial u}
\end{array}\right)_{u=0}=0,
$$

from which we conclude

$$
\left(\begin{array}{l}
\frac{\partial \tau_{1}}{\partial u} \\
\frac{\partial \tau_{2}}{\partial u}
\end{array}\right)_{u=0}=0
$$

in view of (5) and (6). Equation (10) has two important implications.

First, if we set $\gamma=i$, the equation (10) indicates that the local stability crossing curve $\mathcal{T}_{\left(\omega_{0}, \tau_{10}, \tau_{20}\right)}$ may have a cusp at $\left(\tau_{10}, \tau_{20}\right)$ [9]. Indeed, as will be confirmed by considering the second-order derivative in the next section, $\mathcal{T}_{\left(\omega_{0}, \tau_{10}, \tau_{20}\right)}$ partitions a sufficiently small neighborhood of $\left(\tau_{10}, \tau_{20}\right)$ into a great sector (or G-sector) and a small sector ${ }^{1}$ (or S-sector) as shown in Figure 1. We will investigate how the double roots at $i \omega_{0}$ migrate as $\left(\tau_{1}, \tau_{2}\right)$ moves from $\left(\tau_{10}, \tau_{20}\right)$ to the $\mathrm{G}$-sector or the S-sector.

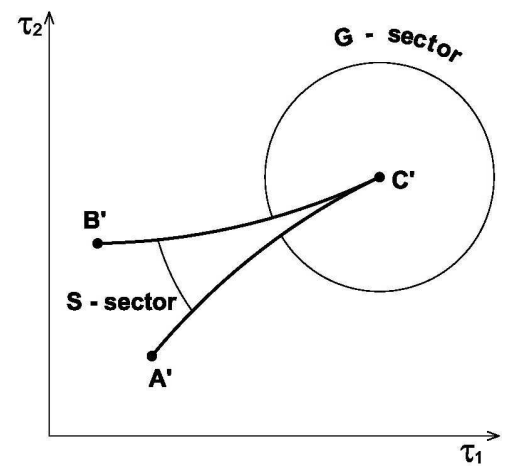

Fig. 1. G-sector and S-sector.

To obtain the second implication, we first show the following.

Lemma 2: Consider $s_{a} \in \mathcal{N}_{\delta}^{\circ}\left(s_{0}\right), \delta>0$ sufficiently small, and let $\tau_{1 a}=\tau_{1}\left(s_{a}\right), \tau_{2 a}=\tau_{2}\left(s_{a}\right)$ as defined in Proposition 1. Then

Proof: Let

$$
\left.\frac{\partial}{\partial s} p\left(s, \tau_{1 a}, \tau_{2 a}\right)\right|_{s=s_{a}} \neq 0 .
$$

$$
s_{a}=s_{0}+u \gamma,|\gamma|=1
$$

then,

$$
\begin{aligned}
& \left.\frac{\partial p}{\partial s}\right|_{\substack{s=s_{a} \\
\tau_{1}=\tau_{1 a} \\
\tau_{2}=\tau_{2 a}}}=\left.\frac{\partial}{\partial s} p(s)\right|_{\substack{s=s_{0} \\
\tau_{1}=\tau_{10} \\
\tau_{2}=\tau_{20}}}+\left.\frac{\partial^{2} p}{\partial s^{2}}\right|_{\substack{s=s_{0} \\
\tau_{1}=\tau_{10} \\
\tau_{2}=\tau_{20}}} \gamma u \\
& +\left.\left.\frac{\partial^{2} p}{\partial s \partial \tau_{1}}\right|_{\substack{s=s_{0} \\
\tau_{1}=\tau_{10} \\
\tau_{2}=\tau_{20}}} \frac{\partial \tau_{1}}{\partial u}\right|_{u=0} u \\
& +\left.\left.\frac{\partial^{2} p}{\partial s \partial \tau_{2}}\right|_{\substack{s=s_{0} \\
\tau_{1}=\tau_{10} \\
\tau_{2}=\tau_{20}}} \frac{\partial \tau_{2}}{\partial u}\right|_{u=0} u+O\left(u^{2}\right) \\
& =0+\left.\frac{\partial^{2} p}{\partial s^{2}}\right|_{\substack{s=s_{0} \\
\tau_{1}=\tau_{10} \\
\tau_{2}=\tau_{20}}} \gamma u+0+0+O\left(u^{2}\right),
\end{aligned}
$$

from which we may conclude (11) in view of (4).

The implicit function theorem allows us to conclude the following from Lemma 2.

Proposition 3: Let $s_{a}, \tau_{1 a}$ and $\tau_{2 a}$ be defined as in Lemma 2. Then there exists a sufficiently small neighborhood of $\left(\tau_{1 a}, \tau_{2 a}\right)$ such that the equation (1) defines a unique function $s\left(\tau_{1}, \tau_{2}\right)$ with the function value restricted in a small neighborhood of $s_{a}$.

The second implication of the equation (10) may be stated as the following corollary, which is a consequence

\footnotetext{
${ }^{1}$ We have used the word "small" in a sense analogous to "small solution": a small sector is contained by a sector with straight sides with arbitrarily small angle when the neighborhood is sufficiently small.
} 
of Propositions 1 and 3.

Corollary 4: Let $s_{a}, \tau_{1 a}$ and $\tau_{2 a}$ be defined as in Lemma 2. Then equation (1) defines a bijection between $s$ in a small neighborhood of $s_{a}$ and $\left(\tau_{1}, \tau_{2}\right)$ in a small neighborhood of $\left(\tau_{1 a}, \tau_{2 a}\right)$.

Obviously, the small neighborhoods referred in Proposition 3 and Corollary 4 above should not include $s_{0}$ and $\left(\tau_{10}, \tau_{20}\right)$ in view of (3). In view of continuity of solutions of (1) with respect to the parameters $\left(\tau_{1}, \tau_{2}\right)$, Corollary 4 may be equivalently stated as follows.

Corollary 5: For all $\left(\tau_{1}, \tau_{2}\right) \in \mathcal{N}_{\varepsilon}^{\circ}\left(\tau_{10}, \tau_{20}\right)$ with $\varepsilon>0$ sufficiently small, the characteristic equation (1) has exactly two simple roots in a small neighborhood of $s_{0}$.

\section{MAPPING IN A NEIGHBORHOOD OF DOUBLE ROOT}

In this section, it will be shown that we can very clearly describe the mapping between " $s$ " and $\left(\tau_{1}, \tau_{2}\right)$ in the neighborhood of " $s_{0}$ " based on the second order derivative when $s-s_{0}$ is restricted to one quadrant. From this description, we may obtain the information on how the double root migrates as $\left(\tau_{1}, \tau_{2}\right)$ moves from $\left(\tau_{10}, \tau_{20}\right)$ to the $\mathbf{G}$-sector or the $\mathbf{S}$ sector in Figure 1 according to the sign of $D$, and whether the positive local stability crossing curve $\mathcal{T}_{\left(\omega_{0}, \tau_{10}, \tau_{20}\right)}^{+}$is on the clockwise side or the counterclockwise side of $\mathcal{T}_{(-1}^{-}$ in the $\mathrm{S}$-sector.

Taking derivative of (9) with respect to the radial variable $u$, we obtain

$$
\begin{array}{r}
\frac{\partial^{2} p}{\partial \tau_{1}^{2}}\left(\frac{\partial \tau_{1}}{\partial u}\right)^{2}+2 \frac{\partial^{2} p}{\partial \tau_{1} \partial \tau_{2}} \frac{\partial \tau_{1}}{\partial u} \frac{\partial \tau_{2}}{\partial u}+2 \frac{\partial^{2} p}{\partial \tau_{1} \partial s} \frac{\partial \tau_{1}}{\partial u} \gamma+ \\
+\frac{\partial p}{\partial \tau_{1}} \frac{\partial^{2} \tau_{1}}{\partial u^{2}}+\frac{\partial^{2} p}{\partial \tau_{2}^{2}}\left(\frac{\partial \tau_{2}}{\partial u}\right)^{2}+2 \frac{\partial^{2} p}{\partial \tau_{2} \partial s} \frac{\partial \tau_{2}}{\partial u} \gamma+ \\
+\frac{\partial p}{\partial \tau_{2}} \frac{\partial^{2} \tau_{2}}{\partial u^{2}}+\frac{\partial^{2} p}{\partial s^{2}} \gamma^{2}=0
\end{array}
$$

Setting $u=0$ and applying (10) in (12), we arrive at

$$
\left[\frac{\partial p}{\partial \tau_{1}} \frac{\partial^{2} \tau_{1}}{\partial u^{2}}+\frac{\partial p}{\partial \tau_{2}} \frac{\partial^{2} \tau_{2}}{\partial u^{2}}+\frac{\partial^{2} p}{\partial s^{2}} \gamma^{2}\right]_{\substack{s=s_{0} \\ \tau_{1}=\tau_{10} \\ \tau_{2}=\tau_{20}}}=0
$$

The above may be solved for $\frac{\partial^{2} \tau_{1}}{\partial u^{2}}$ and $\frac{\partial^{2} \tau_{2}}{\partial u^{2}}$ to obtain,

$$
\begin{aligned}
& \left(\begin{array}{l}
\frac{\partial^{2} \tau_{1}}{\partial u^{2}} \\
\frac{\partial^{2} \tau_{2}}{\partial u^{2}}
\end{array}\right)_{\substack{s=s_{0} \\
\tau_{1}=\tau_{10} \\
\tau_{2}=\tau_{20}}}=
\end{aligned}
$$

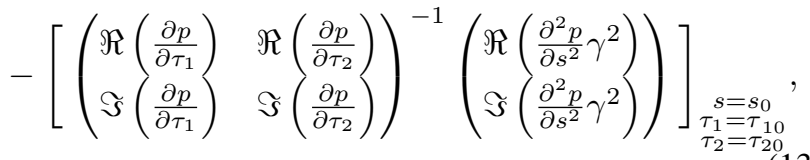

which may also be written in a complex form

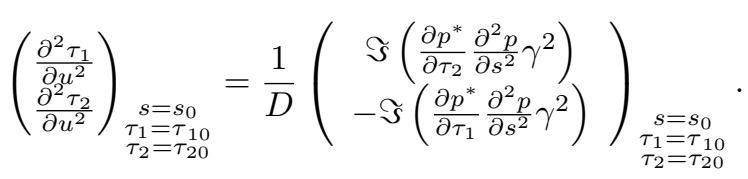

In view of (10), the tangent of the curve describing $\left(\tau_{1}, \tau_{2}\right)$ as a function of $u$ at $\left(\tau_{10}, \tau_{20}\right)$ is determined by the second order derivative given in (13) or (14).

Before proceeding further, it is helpful to recall the following well known fact. It can be found in various elementary books that deal with geometry, see for example [5].

Lemma 6: Let $x^{(0)} \in \mathbb{R}^{2}$ and $M \in \mathbb{R}^{2 \times 2}$ be fixed. For any $x \in \mathbb{R}^{2}$, let $\theta$ be the angle to rotate $x^{(0)}$ to the direction of $x$ in the counterclockwise direction. Let $\phi(\theta)$ be the angle to rotate $M x^{(0)}$ to the direction of $M x$ in the counterclockwise direction if $\operatorname{det}(M)>0$, and in the clockwise direction if $\operatorname{det}(M)<0$. Then the function $\phi(\theta)$ satisfies the following:

i) $\phi(\theta)$ is a continuous and increasing function of $\theta$

ii) $0<\phi(\theta)<\pi$ if and only if $0<\theta<\pi$.

We now make the following two observations about the second order derivative expression (13).

First, set $\gamma=i$ and $\gamma=-i$, the expression determines the tangent of $\mathcal{T}_{\left(\omega_{0}, \tau_{10}, \tau_{20}\right)}$ as $\omega \rightarrow \omega_{0}$ from each side. As

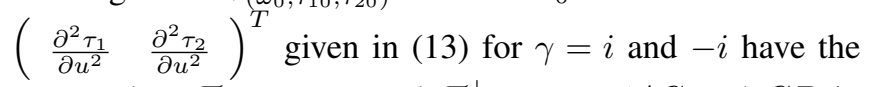
same value, $\mathcal{T}_{\left(\omega_{0}, \tau_{10}, \tau_{20}\right)}^{-}$and $\mathcal{T}_{\left(\omega_{0}, \tau_{10}, \tau_{20}\right)}^{+}(A C$ and $C B$ in Figure 1) are tangent to each other at the point $\left(\tau_{10}, \tau_{20}\right)$, thus forming a cusp.

Second, as $\gamma$ rotates through a $90^{\circ}$ angle in a counterclockwise direction, $\frac{\partial^{2} p}{\partial s^{2}} \gamma^{2}$ rotates through a $180^{\circ}$ angle in

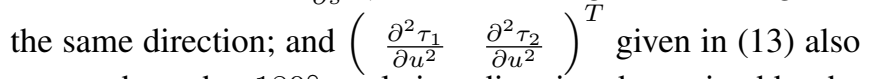
rotates through a $180^{\circ}$ angle in a direction determined by the sign of $D$, which is the determinant of the matrix inverted: the rotation is counterclockwise if $D>0$, and it is clockwise if $D<0$ (according to Lemma 6).

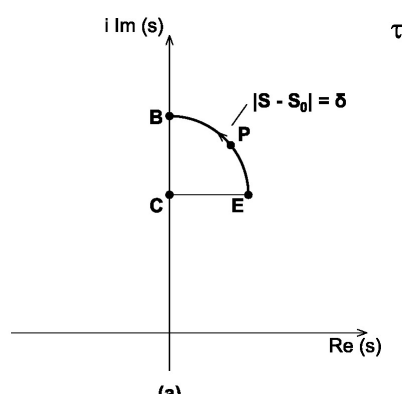

(a)

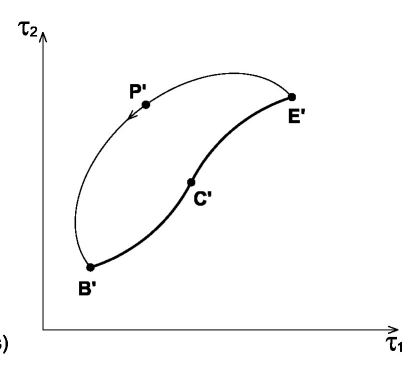

(b)
Fig. 2. The mapping $\left(\tau_{1}(s), \tau_{2}(s)\right)$ with $s-s_{0}$ in the first quadrant.

With the above observations, and the fact that

$$
\left(\begin{array}{c}
\tau_{1}(s) \\
\tau_{2}(s)
\end{array}\right)=\left(\begin{array}{c}
\tau_{10} \\
\tau_{20}
\end{array}\right)+\frac{u^{2}}{2}\left(\begin{array}{c}
\frac{\partial^{2} \tau_{1}}{\partial u^{2}} \\
\frac{\partial^{2} \tau_{2}}{\partial u^{2}}
\end{array}\right)_{\substack{s=s_{0} \\
\tau_{1}=\tau_{10} \\
\tau_{2}=\tau_{20}}}+O\left(u^{3}\right)
$$

we may describe the local mapping $\left(\tau_{1}(s), \tau_{2}(s)\right)$ in a very informative manner when $s-s_{0}$ is restricted to one quadrant. The situation for $s-s_{0}$ in the first quadrant

$$
Q_{1}=\left\{s=s_{0}+u e^{i \theta} \mid 0<u<\delta, 0 \leq \theta \leq \pi / 2\right\}
$$

with $D>0$ is illustrated in Figure 2: the line segment $C E$ (from $s_{0}$ to $s_{0}+\delta$ ) is mapped to the curve $C^{\prime} E^{\prime}$ in the $\tau_{1}-\tau_{2}$ space, the arc $\operatorname{EPB}\left(s=s_{0}+\delta e^{i \theta}, 0 \leq \theta \leq \pi / 2\right)$ is mapped to the curve $E^{\prime} P^{\prime} B^{\prime}$, and the line segment $B C$ (from $s_{0}+\delta i$ to $s_{0}$ ) is mapped to the curve $B^{\prime} C^{\prime}$. In view of the second order derivatives, $B^{\prime} C^{\prime}$ and $C^{\prime} E^{\prime}$ have the same tangent at $C^{\prime}$. Continuity and local bijectivity (Corollary 4 ) imply that the singly connected region bounded by the line segments $B C, C E$ and the arc $E P B$ is mapped by $\left(\tau_{1}(s), \tau_{2}(s)\right)$ bijectively to the singly connected region bounded by the curves $B^{\prime} C^{\prime}, C^{\prime} E^{\prime}$ and $E^{\prime} P^{\prime} B^{\prime}$. 
When $D<0$, the curve $E^{\prime} P^{\prime} B^{\prime}$ is roughly clockwise (instead of counterclockwise as in Figure 2) relative to the point $C^{\prime}$. The mapping with $s-s_{0}$ in the other three quadrants are similar.

The complete mapping $\left(\tau_{1}(s), \tau_{2}(s)\right)$ with $s-s_{0}$ in all four quadrants may be divided into four possible cases depending on the sign of $D$ and whether $\mathcal{T}_{\left(\omega_{0}, \tau_{10}, \tau_{20}\right)}^{-}$is on the counterclockwise or the clockwise side of $\mathcal{T}_{\left(\omega_{0}, \tau_{10}, \tau_{20}\right)}^{+}$in the S-sector. The migration of the double roots in all case is summarized in the following theorem.

Theorem 7 (Migration of Double Roots): If $\left(\tau_{1}, \tau_{2}\right)$ is in the G-sector in a sufficiently small neighborhood of $\left(\tau_{10}, \tau_{20}\right)$, then one root of (1) in the neighborhood of $s_{0}$ is in the right half plane, the other is in the left half plane.

When $\left(\tau_{1}, \tau_{2}\right)$ is in the S-sector, then the two roots are either both in the left half plane or both in the right half plane. Specifically,

Case i. If $D>0$, and $\mathcal{T}_{\left(\omega_{0}, \tau_{10}, \tau_{20}\right)}^{-}$is in the counterclockwise side of $\mathcal{T}_{\left(\omega_{0}, \tau_{10}, \tau_{20}\right)}^{+}$in the $\mathrm{S}$-sector, then both roots are in the left half plane.

Case ii. If $D>0$, and $\mathcal{T}_{\left(\omega_{0}, \tau_{10}, \tau_{20}\right)}^{-}$is in the clockwise side of $\mathcal{T}_{\left(\omega_{0}, \tau_{10}, \tau_{20}\right)}^{+}$in the S-sector, then both roots are in the right half plane.

Case iii. If $D<0$, and $\mathcal{T}_{\left(\omega_{0}, \tau_{10}, \tau_{20}\right)}^{-}$is in the counterclockwise side of $\mathcal{T}_{\left(\omega_{0}, \tau_{10}, \tau_{20}\right)}^{+}$in the $\mathrm{S}$-sector, then both roots are in the right half plane.

Case iv. If $D<0$, and $\mathcal{T}_{\left(\omega_{0}, \tau_{10}, \tau_{20}\right)}^{-}$is in the clockwise side of $\mathcal{T}_{\left(\omega_{0}, \tau_{10}, \tau_{20}\right)}^{+}$in the $S$-sector, then both roots are in the left half plane.

Proof: Consider case i. The situation is illustrated in Figure 3. Let the region bounded by the arc EPB and line segments $B C$ and $C E$ be denoted as $I$, and the region bounded by the curves $E^{\prime} P^{\prime} B^{\prime}, B^{\prime} C^{\prime}$ and $C^{\prime} E^{\prime}$ be denoted as $I^{\prime}$. Similarly, region $I I$ is bounded by $B Q F, F C, C B$, and region $I I^{\prime}$ is bounded by $B^{\prime} Q^{\prime} F^{\prime}, F^{\prime} C^{\prime}, C^{\prime} B^{\prime}$; region $I I I$ is bounded by FRA,AC,CF, and $I I I^{\prime}$ is bounded by $F^{\prime} R^{\prime} A^{\prime}, A^{\prime} C^{\prime}, C^{\prime} F^{\prime}$; region $I V$ is bounded by $A S E$, $E C, C A$, and region $I V^{\prime}$ is bounded by $A^{\prime} S^{\prime} E^{\prime}, E^{\prime} C^{\prime}$, $C^{\prime} A^{\prime}$. As discussed before the theorem, $\left(\tau_{1}(s), \tau_{2}(s)\right)$ is a bijection from $I$ to $I^{\prime}$ when $s$ is restricted to $I$. Similarly, $\left(\tau_{1}(s), \tau_{2}(s)\right)$ is a bijection from $I I$ to $I I^{\prime}$ when restricted to $I I$, or from $I I I$ to $I I I^{\prime}$ when restricted to $I I I^{\prime}$, or from $I V$ to $I V^{\prime}$ when restricted to $I V$. As the S-sector (in a sufficiently small neighborhood) is contained in $I I^{\prime} \cap I I I^{\prime}$, we may conclude that for any $\left(\tau_{1}, \tau_{2}\right)$ in the S-sector, one of the two characteristic roots in the neighborhood of $s_{0}$ must be in region $I I$, the other must be in region $I I I$, and obviously both in the left half plane. Similarly, the Gsector (in a sufficiently small neighborhood) is contained in $\left(I^{\prime} \cup I V^{\prime}\right) \cap\left(I I^{\prime} \cup I V^{\prime}\right)$. Therefore, for any $\left(\tau_{1}, \tau_{2}\right)$ in the Gsector, one of the two characteristic roots in the neighborhood of $s_{0}$ must be in $I \cup I V$ (in the right half plane), and the other must be in $I I \cup I I I$ (in the left half plane).

Case ii is illustrated in Figures 4. In this case, the S-sector is contained in $I^{\prime} \cap I V^{\prime}$, and therefore, the two characteristic roots in the neighborhood of $s_{0}$ must be in regions $I$ and $I V$, both in the right half plane. The G-sector can still be expressed as $\left(I^{\prime} \cup I V^{\prime}\right) \cap\left(I I^{\prime} \cup I V^{\prime}\right)$.

Case iii is illustrated in Figure 5, and case iv is illustrated in Figure 6, and the conclusions can be drawn in a similar manner.
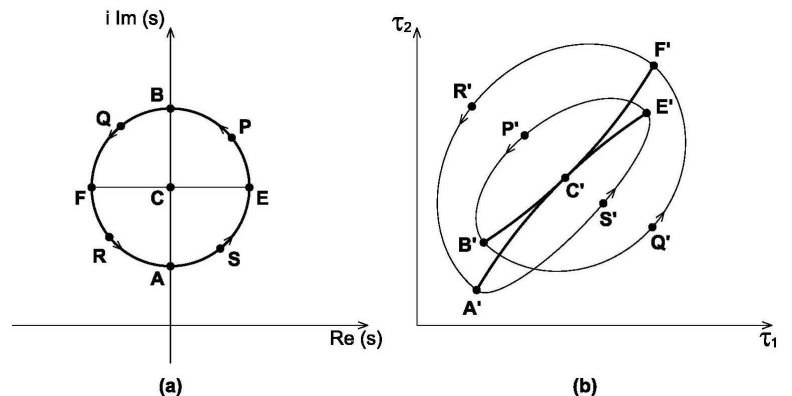

(b)

Fig. 3. The mapping $\left(\tau_{1}(s), \tau_{2}(s)\right)$ in a neighborhood of $s_{0}$. Case i: $D>0$, and $\mathcal{T}_{\left(\omega_{0}, \tau_{10}, \tau_{20}\right)}^{-}$is on the counterclockwise side of $\mathcal{T}_{\left(\omega_{0}, \tau_{10}, \tau_{20}\right)}^{+}$ in the S-sector.

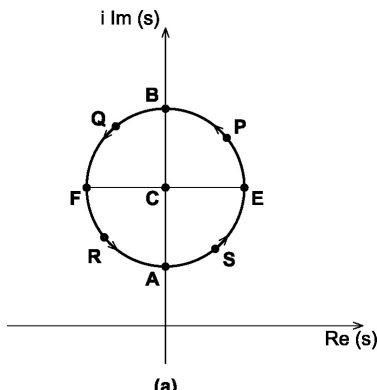

(a)

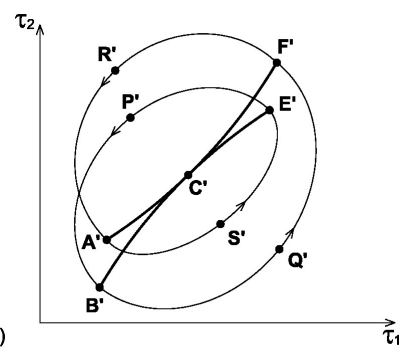

(b)
Fig. 4. The mapping $\left(\tau_{1}(s), \tau_{2}(s)\right)$ in a neighborhood of $s_{0}$. Case ii: $D>0$, and $\mathcal{T}_{\left(\omega_{0}, \tau_{10}, \tau_{20}\right)}^{-}$is on the clockwise side of $\mathcal{T}_{\left(\omega_{0}, \tau_{10}, \tau_{20}\right)}^{+}$in the S-sector.

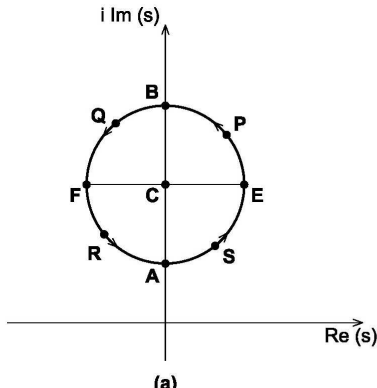

(a)

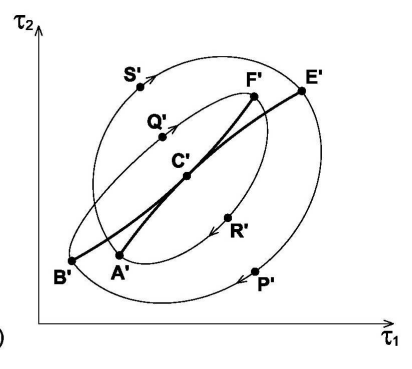

(b)
Fig. 5. The mapping $\left(\tau_{1}(s), \tau_{2}(s)\right)$ in a neighborhood of $s_{0}$. Case iii: $D<0$, and $\mathcal{T}_{\left(\omega_{0}, \tau_{10}, \tau_{20}\right)}^{-}$is on the counterclockwise side of $\mathcal{T}_{\left(\omega_{0}, \tau_{10}, \tau_{20}\right)}^{+}$ in the $\mathrm{S}$-sector.

\section{Algebraic S-SECTOR CONDition AND GLOBAL PERSPECTIVES}

Theorem 7 indicates that the migration pattern of the two roots in the G-sector is always the same for the least degenerate case discussed in this article. However, judging the migration pattern of the two roots in the S-sector requires knowing the sign of $D$ and which side of $\mathcal{T}_{\left(\omega_{0}, \tau_{10}, \tau_{20}\right)}^{+}$ the curve $\mathcal{T}_{\left(\omega_{0}, \tau_{10}, \tau_{20}\right)}^{-}$is in the S-sector. Fortunately, by considering the third order derivatives, an explicit algebraic condition is possible.

Corollary 8 (S-sector Criterion): If $\left(\tau_{1}, \tau_{2}\right)$ is in the Ssector in a sufficiently small neighborhood of $\left(\tau_{10}, \tau_{20}\right)$, then 

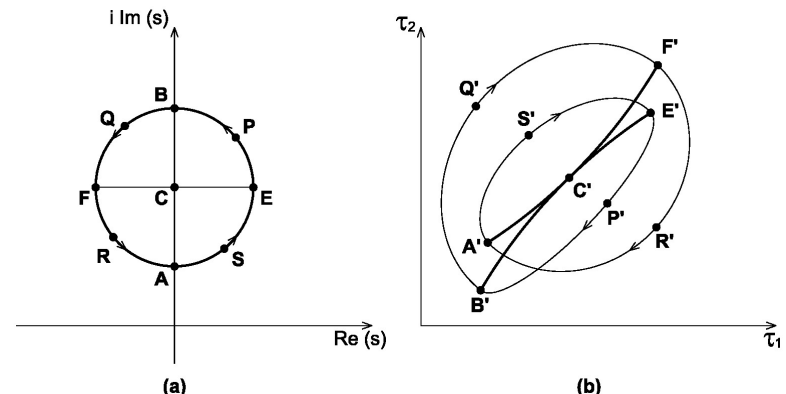

(b)

Fig. 6. The mapping $\left(\tau_{1}(s), \tau_{2}(s)\right)$ in a neighborhood of $s_{0}$. Case iv: $D<0$, and $\mathcal{T}_{\left(\omega_{0}, \tau_{10}, \tau_{20}\right)}^{-}$is on the clockwise side of $\mathcal{T}_{\left(\omega_{0}, \tau_{10}, \tau_{20}\right)}^{+}$in the $\mathrm{S}$-sector.

the two characteristic roots in the neighborhood of $s_{0}$ are both in the left half plane if

$$
\kappa<0
$$

where

$\kappa=$

$\Re\left[\frac{\partial^{2} p}{\partial s^{2}}\left(-\frac{\partial^{3} p}{\partial s^{3}}+3 \frac{\partial^{2} p}{\partial \tau_{1} \partial s} \frac{\partial^{2} \tau_{1}}{\partial u^{2}}+3 \frac{\partial^{2} p}{\partial \tau_{2} \partial s} \frac{\partial^{2} \tau_{2}}{\partial u^{2}}\right)\right]_{\substack{s=s_{0} \\ \tau_{1}=\tau_{10}=\tau_{20} \\ \gamma=i}}$,

and $\frac{\partial^{2} \tau_{j}}{\partial u^{2}}, j=1,2$ are evaluated by (14) or (13) with $\gamma=i$. If

$$
\kappa>0
$$

instead, then both roots are in the right half plane.

Proof: Differentiate (12) with respect to $u$, we obtain

$$
\begin{array}{r}
\frac{\partial^{3} p}{\partial \tau_{1}^{3}}\left(\frac{\partial \tau_{1}}{\partial u}\right)^{3}+3 \frac{\partial^{3} p}{\partial \tau_{1}^{2} \tau_{2}}\left(\frac{\partial \tau_{1}}{\partial u}\right)^{2} \frac{\partial \tau_{2}}{\partial u}+ \\
+3 \frac{\partial^{3} p}{\partial \tau_{1}^{2} \partial s}\left(\frac{\partial \tau_{1}}{\partial u}\right)^{2} \gamma+3 \frac{\partial^{2} p}{\partial \tau_{1}^{2}} \frac{\partial \tau_{1}}{\partial u} \frac{\partial^{2} \tau_{1}}{\partial u^{2}}+ \\
+3 \frac{\partial^{3} p}{\partial \tau_{1} \partial \tau_{2}^{2}} \frac{\partial \tau_{1}}{\partial u}\left(\frac{\partial \tau_{2}}{\partial u}\right)^{2}+ \\
+6 \frac{\partial^{3} p}{\partial \tau_{1} \partial \tau_{2} \partial s} \frac{\partial \tau_{1}}{\partial u} \frac{\partial \tau_{2}}{\partial u} \gamma+3 \frac{\partial^{2} p}{\partial \tau_{1} \partial \tau_{2}} \frac{\partial^{2} \tau_{1}}{\partial u^{2}} \frac{\partial \tau_{2}}{\partial u}+ \\
+3 \frac{\partial^{2} p}{\partial \tau_{1} \partial \tau_{2}} \frac{\partial \tau_{1}}{\partial u} \frac{\partial^{2} \tau_{2}}{\partial u^{2}}+3 \frac{\partial^{3} p}{\partial \tau_{1} \partial s^{2}} \frac{\partial \tau_{1}}{\partial u} \gamma^{2}+ \\
+3 \frac{\partial^{2} p}{\partial \tau_{1} \partial s} \frac{\partial^{2} \tau_{1}}{\partial u^{2}} \gamma+\frac{\partial p}{\partial \tau_{1}} \frac{\partial^{3} \tau_{1}}{\partial u^{3}}+\frac{\partial^{3} p}{\partial \tau_{2}^{3}}\left(\frac{\partial \tau_{2}}{\partial u}\right)^{3}+ \\
+3 \frac{\partial^{2} p}{\partial \tau_{2} \partial s} \frac{\partial^{2} \tau_{2}}{\partial u^{2}} \gamma+\frac{\partial p}{\partial \tau_{2}} \frac{\partial^{3} \tau_{2}}{\partial u^{3}}+\frac{\partial^{3} p}{\partial s^{3}} \gamma^{3}=0 .
\end{array}
$$

Setting $u=0$ and using (10) in the above yields

$$
\begin{array}{r}
{\left[3 \frac{\partial^{2} p}{\partial \tau_{1} \partial s} \frac{\partial^{2} \tau_{1}}{\partial u^{2}} \gamma+\frac{\partial p}{\partial \tau_{1}} \frac{\partial^{3} \tau_{1}}{\partial u^{3}}+3 \frac{\partial^{2} p}{\partial \tau_{2} \partial s} \frac{\partial^{2} \tau_{2}}{\partial u^{2}} \gamma+\right.} \\
\left.+\frac{\partial p}{\partial \tau_{2}} \frac{\partial^{3} \tau_{2}}{\partial u^{3}}+\frac{\partial^{3} p}{\partial s^{3}} \gamma^{3}\right]_{\substack{s=s_{0} \\
\tau_{1}=\tau_{10} \\
\tau_{2}=\tau_{20}}}=0
\end{array}
$$

which can be solved to obtain

$$
\begin{aligned}
& \left(\begin{array}{l}
\frac{\partial^{3} \tau_{1}}{\partial u^{3}} \\
\frac{\partial^{3} \tau_{2}}{\partial u^{3}}
\end{array}\right)_{\substack{s=s_{0} \\
\tau_{1}=\tau_{10} \\
\tau_{2}=\tau_{20}}}= \\
& -\left(\begin{array}{ll}
\Re\left(\frac{\partial p}{\partial \tau_{1}}\right) & \Re\left(\frac{\partial p}{\partial \tau_{2}}\right) \\
\Im\left(\frac{\partial p}{\partial \tau_{1}}\right) & \Im\left(\frac{\partial p}{\partial \tau_{2}}\right)
\end{array}\right) \underbrace{-1}_{\substack{s=s_{0} \\
\begin{array}{c}
\tau_{1}=\tau_{10} \\
\tau_{2}=\tau_{20}
\end{array}}}\left(\begin{array}{l}
\Re(B) \\
\Im(B)
\end{array}\right),
\end{aligned}
$$

where

$$
\begin{aligned}
B & =\left[\frac{\partial^{3} p}{\partial s^{3}} \gamma^{3}+3 \frac{\partial^{2} p}{\partial \tau_{1} \partial s} \frac{\partial^{2} \tau_{1}}{\partial u^{2}} \gamma\right. \\
& \left.+3 \frac{\partial^{2} p}{\partial \tau_{2} \partial s} \frac{\partial^{2} \tau_{2}}{\partial u^{2}} \gamma\right]_{\begin{array}{c}
\text { s } \\
s=s_{0} \\
\tau_{2}=\tau_{10} \\
\tau_{2}=\tau_{20}
\end{array}} .
\end{aligned}
$$

Let

$$
\left(\begin{array}{l}
\frac{\partial^{k} \tau_{1}}{\partial u^{k}} \\
\frac{\partial^{k} \tau_{2}}{\partial u^{k}}
\end{array}\right)_{ \pm}=\left(\begin{array}{c}
\frac{\partial^{k} \tau_{1}}{\partial u^{k}} \\
\frac{\partial^{k} \tau_{2}}{\partial u^{k}}
\end{array}\right)_{\substack{s=s_{0} \\
\tau_{1}=\tau_{10} \\
\tau_{2}=\tau_{20} \\
\gamma= \pm i}}, k=1,2,3
$$

and

$$
\left(\begin{array}{l}
\tau_{1} \\
\tau_{2}
\end{array}\right)_{ \pm}=\left(\begin{array}{c}
\tau_{1}\left(s_{0} \pm \delta i\right) \\
\tau_{2}\left(s_{0} \pm \delta i\right)
\end{array}\right)
$$

Then Taylor series gives

$$
\begin{gathered}
\left(\begin{array}{c}
\tau_{1} \\
\tau_{2}
\end{array}\right)_{ \pm}=\left(\begin{array}{l}
\tau_{10} \\
\tau_{20}
\end{array}\right)+\delta\left(\begin{array}{c}
\frac{\partial \tau_{1}}{\partial u} \\
\frac{\partial \tau_{2}}{\partial u}
\end{array}\right)_{ \pm} \\
+\frac{\delta^{2}}{2}\left(\begin{array}{c}
\frac{\partial^{2} \tau_{1}}{\partial u^{2}} \\
\frac{\partial^{2} \tau_{2}}{\partial u^{2}}
\end{array}\right)_{ \pm}+\frac{\delta^{3}}{6}\left(\begin{array}{c}
\frac{\partial^{3} \tau_{1}}{\partial u^{3}} \\
\frac{\partial^{3} \tau_{2}}{\partial u^{3}}
\end{array}\right)_{ \pm}+O\left(\delta^{4}\right) .
\end{gathered}
$$

But according to (10) and (13), we have

$$
\begin{aligned}
& \left(\begin{array}{l}
\frac{\partial \tau_{1}}{\partial u} \\
\frac{\partial \tau_{2}}{\partial u}
\end{array}\right)_{ \pm}=0 \\
& \left(\begin{array}{l}
\frac{\partial^{2} \tau_{1}}{\partial u^{2}} \\
\frac{\partial^{2} \tau_{2}}{\partial u^{2}}
\end{array}\right)_{+}=\left(\begin{array}{l}
\frac{\partial^{2} \tau_{1}}{\partial u^{2}} \\
\frac{\partial^{2} \tau_{2}}{\partial u^{2}}
\end{array}\right)_{-} .
\end{aligned}
$$

Therefore,

$$
\begin{aligned}
& \left(\begin{array}{c}
\Delta \tau_{1} \\
\Delta \tau_{2}
\end{array}\right) \triangleq\left(\begin{array}{l}
\tau_{1} \\
\tau_{2}
\end{array}\right)_{+}-\left(\begin{array}{c}
\tau_{1} \\
\tau_{2}
\end{array}\right)_{-} \\
& =\frac{\delta^{3}}{6}\left[\left(\begin{array}{c}
\frac{\partial^{3} \tau_{1}}{\partial u^{3}} \\
\frac{\partial^{3} \tau_{2}}{\partial u^{3}}
\end{array}\right)_{+}-\left(\begin{array}{c}
\frac{\partial^{3} \tau_{1}}{\partial u^{3}} \\
\frac{\partial^{3} \tau_{2}}{\partial u^{3}}
\end{array}\right)_{-}\right]+O\left(\delta^{4}\right) . \\
& =-\frac{\delta^{3}}{6}\left(\begin{array}{ll}
\Re\left(\frac{\partial p}{\partial \tau_{1}}\right) & \Re\left(\frac{\partial p}{\partial \tau_{2}}\right) \\
\Im\left(\frac{\partial p}{\partial \tau_{1}}\right) & \Im\left(\frac{\partial p}{\partial \tau_{2}}\right)
\end{array}\right) \begin{array}{c}
-1 \\
\begin{array}{c}
\Re(\Delta B) \\
s=s_{0} \\
\tau_{1}=\tau_{10} \\
\tau_{2}=\tau_{20}
\end{array}
\end{array}\left(\begin{array}{c}
\Im(\Delta B) \\
\Im(\Delta B)
\end{array}\right) \\
& +O\left(\delta^{4}\right)
\end{aligned}
$$

where

$$
\begin{gathered}
\Delta B=\left.B\right|_{\gamma=i}-\left.B\right|_{\gamma=-i} \\
=2 i\left[-\frac{\partial^{3} p}{\partial s^{3}}+3 \frac{\partial^{2} p}{\partial \tau_{1} \partial s}\left(\frac{\partial^{2} \tau_{1}}{\partial u^{2}}\right)_{+}+3 \frac{\partial^{2} p}{\partial \tau_{2} \partial s}\left(\frac{\partial^{2} \tau_{2}}{\partial u^{2}}\right)_{+}\right]_{\substack{s=s_{0} \\
\tau_{1}=\tau_{10} \\
\tau_{2}=\tau_{20}}}
\end{gathered}
$$

As the tangent direction of the local stability crossing curve 
$\mathcal{T}_{\left(\omega_{0}, \tau_{10}, \tau_{20}\right)}$ at the cusp $\left(\tau_{10}, \tau_{20}\right)$ is $\left(\frac{\partial^{2} \tau_{1}}{\partial u^{2}}, \frac{\partial^{2} \tau_{2}}{\partial u^{2}}\right)_{+}^{T}$, it can be easily seen that the $\mathcal{T}_{\left(\omega_{0}, \tau_{10}, \tau_{20}\right)}^{-}$is in the counterclockwise side of $\mathcal{T}_{\left(\omega_{0}, \tau_{10}, \tau_{20}\right)}^{+}$if we may reach the direction of $\left(\frac{\partial^{2} \tau_{1}}{\partial u^{2}}, \frac{\partial^{2} \tau_{2}}{\partial u^{2}}\right)_{+}^{T}$ by rotating $\left(\Delta \tau_{1}, \Delta \tau_{2}\right)$ counterclockwise through an angle $\theta \in(0, \pi)$ as is shown in Figure 7 . Let $\left(-\frac{\partial^{2} p}{\partial s^{2}}\right)_{0}=\left(-\frac{\partial^{2} p}{\partial s^{2}}\right)_{\substack{s=s_{0} \\ \tau_{1}=\tau_{10} \\ \tau_{2}=\tau_{20}}}$. Comparing the expressions (20) and (13) and using Lemma 6, we can see that the above can be achieved if we can reach the direction of $\left(-\frac{\partial^{2} p}{\partial s^{2}}\right)$ by rotating $\Delta B$ counterclockwise through an angle of $\theta \in(0, \pi)$ if $D>0$ (which is case $\mathrm{i}$ in Theorem 7). The rotation from $\Delta B$ to $\left(-\frac{\partial^{2} p}{\partial s^{2}}\right)$ needs to be clockwise if $D<0$ (which is case iii). The counterclockwise rotation from $\Delta B$ to $\left(-\frac{\partial^{2} p}{\partial s^{2}}\right)_{0}$ may be expressed as

$$
\Re(\Delta B) \Im\left(-\frac{\partial^{2} p}{\partial s^{2}}\right)_{0}-\Im(\Delta B) \Re\left(-\frac{\partial^{2} p}{\partial s^{2}}\right)_{0}>0,
$$

which is equivalent to (15), and the conclusion is valid in this case in view of case $\mathrm{i}$ in Theorem 7 . It can be similarly shown that if we can rotate $\Delta B$ to the direction of $\left(-\frac{\partial^{2} p}{\partial s^{2}}\right)$ clockwise through an angle of $\theta \in(0, \pi)$, then (16) is satisfied, and the conclusion is valid in this case also in view of case iii in Theorem 7.

Similarly, we can show that $\kappa>0$ and $D>0$, or $\kappa<0$ and $D<0$ can guarantee that we can reach the direction of $\left(\frac{\partial^{2} \tau_{1}}{\partial u^{2}}, \frac{\partial^{2} \tau_{2}}{\partial u^{2}}\right)_{+}^{T}$ by rotating $\left(\Delta \tau_{1}, \Delta \tau_{2}\right)$ clockwise through an angle $\theta \in(0, \pi)$, and the conclusions are true in view of case ii and case iv in Theorem 7 . We have exhausted all possibilities, and the corollary is proven.

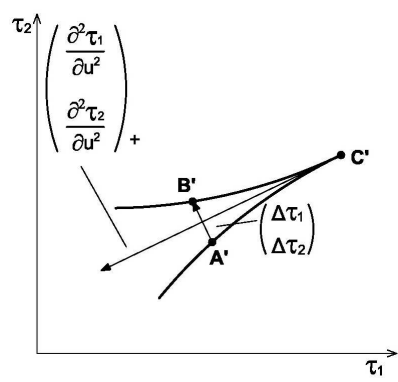

Fig. 7. $\mathcal{T}_{\left(\omega_{0}, \tau_{10}, \tau_{20}\right)}^{-}$is on the counterclockwise side of $\mathcal{T}_{\left(\omega_{0}, \tau_{10}, \tau_{20}\right)}^{+}$, and the angle $\phi$ needed to rotate $A^{\prime} B^{\prime}$ to the direction of $\left(\frac{\partial^{2} \tau_{1}}{\partial u^{2}}, \frac{\partial^{2} \tau_{2}}{\partial u^{2}}\right)_{\gamma=i}^{T}$ must satisfy $0<\phi<\pi$.

If $\kappa=0$, higher order derivatives may be used to evaluate conditions in Theorem 7.

It should be noticed that the roots of the characteristics discussed in Theorem 7 and Corollary 8 are restricted to the neighborhood of $s_{0}=j \omega_{0}$. Because characteristic roots are distributed symmetrically with respect to the real axis, there is also a double root at $s_{0}^{*}=-j \omega_{0}$ when $\tau_{1}=\tau_{10}, \tau_{2}=\tau_{20}$. When $\left(\tau_{1}, \tau_{2}\right)$ deviates from $\left(\tau_{10}, \tau_{20}\right)$, the migration of the two roots in the neighborhood of $s_{0}^{*}$ follows the same pattern as those in the neighborhood of $s_{0}$.
There may also be roots on the imaginary axis outside of the neighborhoods of $s_{0}$ and $s_{0}^{*}$. The migration of these imaginary roots need to be analyzed separately.

Finally, the roots on the right half plane remain on the right half plane as long as $\left(\tau_{1}, \tau_{2}\right)$ stay within a sufficiently small neighborhood of $\left(\tau_{10}, \tau_{20}\right)$. Similarly, the roots on the left half plane remain on the left half plane when the deviation of $\left(\tau_{1}, \tau_{2}\right)$ is sufficiently small.

\section{CONCLUSIONS}

The migration pattern of a double characteristic root can be studied without using the Puiseux series in the "least degenerate" case. The local stability crossing curve has a cusp, and divides the neighborhood of the critical point into a G-sector and an S-sector in the delay parameter space. As the delay parameter pair moves to the G-sector, one root moves to the left half plane and the other moves to the right half plane. If the delay parameter pair moves to the S-sector, a simple algebraic criterion may be used to judge whether both roots move to the right half plane or the left half plane.

\section{ACKNOWLEDGEMENT}

Part of the work was completed while Keqin $\mathrm{Gu}$ was visiting L2S. The financial support for this visit was provided by the RTRA Digiteo network in Paris-Saclay area and Southern Illinois University Edwardsville.

\section{REFERENCES}

[1] N. G. Chebotarev and N. N. Meiman, "The Routh-Hurwitz problem for polynomials and for entire functions (in Russian)," Trudy Matem. Inst. Steklov, vol. 26, pp. 1-332, 1949.

[2] J. Chen, P. Fu, S.I. Niculescu, and Z. Guan, "An eigenvalue perturbation approach to stability analysis, part 1: eigenvalue series of matrix operators," SIAM Journal of Control Optimization, 48(8), 5564-5582, 2010

[3] J. Chen, P. Fu, S.I. Niculescu, and Z. Guan, "An eigenvalue perturbation approach to stability analysis, part 2: when will zeros of time-delay systems cross imaginary axis?" SIAM Journal of Control Optimization, 48(8), 5583-5605, 2010.

[4] L. E. El'Sgol'ts and S. B. Norkin, Introduction to the Theory and Application of Differential Equations with Deviating Arguments, Translated by J. L. Casti, Academic Press, New York, 1973.

[5] O. Gonzalez and A. M. Stuart, A First Course in Continuum Mechanics, Cambridge University Press, 2008.

[6] E. N. Gryazina, B. T. Polyak, and A. A. Tremba, "D-decomposition technique state-of-the-art," Automation and Remote Control, vol. 69, no. 12, pp. 1991-2026, 2008.

[7] K. Gu, S.-I. Niculescu, and J. Chen, "On stability of crossing curves for general systems with two delays," J. Math. Anal. Appl., vol. 311 , pp. 231-253, 2005

[8] K. Gu and X. Zheng, X, "Stability crossing set for systems with three scalar delay channels," International Journal of Dynamics and Control, Vol. 2, Issue 2, pp. 164-197, 2014

[9] H. W. Guggenheimer, Differential Geometry, New York: Dover, 1977.

[10] E. Jarlebring and W. Michiels, "Invariance properties in the root sensitivity of time-delay systems with double imaginary roots", Automatica, vol. 46 , no. 6 , pp. $1112-1115,2010$.

[11] T. Kato, Perturbation Theory for Linear Operators, 2nd Ed, SpringerVerlag, Berlin, 1980.

[12] K. Knopp, Theory of Functions, Parts I and II, Translated to English by F. Bagemihl, Dover, Mineola, NY, 1996.

[13] M. S. Lee and C. S. Hsu, "On the $\tau$-decomposition method of stability analysis for retarded dynamical systems," SIAM J. Control, 7:249259, 1969

[14] X.-G. Li, S.-I. Niculescu, A. Çela, H.-H. Wang, and T.-Y. Cai, "On computing Puiseux series for multiple imaginary characteristic roots of LTI systems with commensurate delays," IEEE Trans. Autom. Control, 58, 1338-1343, 2013.

[15] W. Michiels and S.-I. Niculescu, Stability and stabilization of timedelay systems. An eigenvalue based approach, SIAM: Philadelphia, USA, Advances in design and control, vol. 12, 2007. 